Théologiques

Théologiques

\title{
Les victimes d'abus sexuels confrontées à la souffrance et à la violence de la Passion
}

\author{
Jean-Guy Nadeau, Carole Golding et Claude Rochon
}

Volume 13, numéro 2, automne 2005

Violence et souffrance rédemptrices

URI : https://id.erudit.org/iderudit/013606ar

DOI : https://doi.org/10.7202/013606ar

Aller au sommaire du numéro

\section{Éditeur(s)}

Faculté de théologie et de sciences des religions, Université de Montréal

ISSN

1188-7109 (imprimé)

1492-1413 (numérique)

Découvrir la revue

Citer cet article

Nadeau, J.-G., Golding, C. \& Rochon, C. (2005). Les victimes d'abus sexuels confrontées à la souffrance et à la violence de la Passion. Théologiques, 13(2), 83-107. https://doi.org/10.7202/013606ar
Résumé de l'article

Les croyances religieuses offrent en principe des ressources pour faire face aux événements traumatisants, mais il y a aussi des cas où elle en accroît le traumatisme ou en retarde la résolution. L'article rend compte de l'expérience de victimes d'abus sexuels durant l'enfance face à la Passion de Jésus. La réflexion s'organise autour des pôles de l'identification et de l'imitation de la figure de Jésus. Non seulement le discours traditionnel chrétien peut-il s'avérer délétère pour les victimes, mais les nombreuses approches critiques développées au vingtième siècle s'avèrent fréquemment insuffisantes pour les victimes. Il demeure donc impératif de développer une christologie qui refuse le statut de volonté divine à la souffrance et à la violence dont elle est souvent issue.
Tous droits réservés $@$ Faculté de théologie et de sciences des religions, Université de Montréal, 2006
Ce document est protégé par la loi sur le droit d'auteur. L'utilisation des services d'Érudit (y compris la reproduction) est assujettie à sa politique d'utilisation que vous pouvez consulter en ligne.

https://apropos.erudit.org/fr/usagers/politique-dutilisation/ 


\title{
Les victimes d'abus sexuels confrontées à la souffrance et à la violence de la Passion
}

\author{
Jean-Guy Nadeau, Carole Golding et Claude Rochon \\ Faculté de théologie et de sciences des religions \\ Université de Montréal ${ }^{1}$
}

La Passion de Jésus constitue pour la foi chrétienne une ressource majeure pour faire face à la souffrance, voire à la violence, tout en étant elle-même récit de souffrance et de violence. Or, pour les chrétiens et chrétiennes, la Passion est lue à travers les filtres de l'éducation religieuse, de la ritualité et de la culture. "La Passion", en effet, ce n'est pas seulement le texte biblique, mais une figure qui émerge d'un parcours complexe à travers l'expérience comme à travers la culture séculière ou religieuse des croyants: liturgie, iconographie, sermons, discours théologiques et catéchétiques, productions cinématographiques, autorité parentale, drames familiaux qui en ont construit et en disent le sens. Le film controversé, mais extrêmement populaire de Mel Gibson, La Passion du Christ (2004), témoigne éloquemment de ce phénomène, comme le font depuis longtemps les traces du «Minuit chrétien» dans la mémoire des Québécois.

Parmi les thèmes qui dessinent la figure habituelle de la Passion, on retrouve:

- la violence faite à Jésus: crachats, coups de fouets, couronne d'épines, chemin de la croix, dénudation publique, enfoncement des clous, pendaison sur la croix;

- la mort ignoble et douloureuse de Jésus en croix comme sacrifice nécessaire pour notre salut;

- la valeur rédemptrice de la mort et des souffrances de Jésus;

1. Cette recherche a bénéficié d'une subvention du Conseil de recherches en sciences humaines du Canada que nous remercions pour son soutien. 
- le fait que la croix s'inscrit dans le plan de Dieu, voire dans sa vengeance et son amour;

- la soumission de Jésus à la volonté de son Père: «Père, [...] que ta volonté soit faite, et non la mienne» (Lc 22,42);

- la résurrection comme sanction positive de l'obéissance et du sacrifice de Jésus;

- la question de Jésus mourant: «Mon Dieu, mon Dieu, pourquoi m'as-tu abandonné? » (Mt 27,46), question par ailleurs nuancée par «Père, entre tes mains je remets mon esprit.» (Lc 23,46);

- nos péchés comme cause de la mort et des souffrances atroces de Jésus.

Les manifestations de compassion sont loin d'occuper une place majeure dans la Passion qui est bien davantage lieu d'injustice, de violence et d'abandon. Les victimes peuvent y retrouver leur expérience d'injustice, de violence et d'abandon. Le malheur, c'est que certains des motifs de la Passion sont à double tranchant et font parfois problème, bien que ou justement parce que la souffrance y trouve valeur — souffrance rédemptrice, souffrance sauvée! Parmi ces thèmes, qui peuvent aujourd'hui s'avérer aussi bien délétères que salvifiques, se démarquent le sens et la valeur de la souffrance, le sacrifice et la soumission de la victime, le péché et le pardon. De plus, cette souffrance rédemptrice est le fait de la violence des bourreaux auxquels la victime paraît soumise. Certes, la résurrection renverse le signe de la victoire, mais est-il encore nécessaire d'attribuer à la volonté divine une souffrance qui est le fruit de la violence humaine? Si on a longtemps pourchassé les Juifs, arguant qu'ils avaient condamné le Christ à la mort de la croix, n'était-ce pas au détriment d'une théo-logique selon laquelle la croix était en réalité l'instrument de Dieu pour notre salut. Une logique qui fait bien l'affaire des bourreaux! Car si la croix se conjugue avec souffrance, puis avec salut, elle se conjugue d'abord avec violence et agression. Ce qui en fait la valeur, mais peut aussi porter à confusion, surtout lorsqu'on l'attribue à la volonté de Dieu, Seigneur de l'histoire.

Dans un texte particulièrement abrasif, Brown et Parker voient dans la sotériologie classique une théologie abusive qui glorifie la souffrance:

Christianity is an abusive theology that glorifies suffering. [...] the predominant image or theology of the culture is of «divine child abuse " — God the Father demanding and carrying out the suffering and death of his own son? If Christianity is to be liberating for the oppressed, it must itself be liberated from this theology. We must do away with the atonement, this idea 
of a blood sin upon the whole human race which can be washed away only by the blood of the lamb. This bloodthirsty God is the God of the patriarchy who at the moment controls the whole Judeo-Christian tradition. (Brown et Parker 1989, 26)

Citant Mary Daly, elles rappellent que «The qualities that Christianity idealizes, especially for women, are also those of a victim: sacrificial love, passive acceptance of suffering, humility, meekness, etc. Since these are the qualities idealized in Jesus "who died for our sins," his functioning as a model reinforces the scapegoat syndrome for women.» (2) Ivone Gebara signale aussi le rôle d'une théologie instrumentale de la croix et de la Passion dans l'acculturation des femmes à accepter l'abus à la suite du Serviteur souffrant $(1999,122-132)$. Même constat du côté des études sur les abus sexuels: "In an atmosphere which glorifies suffering, females tend to see the [sexual] abuse as their cross to bear, as their way of identifying with the sufferings of the Christ. » (Heggen 1993, 94)

La figure de la Passion paraît particulièrement délicate dans des contextes de victimisation, dont celui des abus sexuels en milieu familial, un phénomène qui touche une large partie de la population. De 25 à $53 \%$ des femmes et de 12 à $31 \%$ des hommes en Occident (les chiffres varient selon l'extension des définitions et les méthodes d'enquête) ont été victimes d'agression sexuelle, dans $80 \%$ des cas avant l'âge de 18 ans et le plus souvent en contexte familial ${ }^{2}$.

Tout abus peut ralentir ou même bloquer le développement spirituel de la victime (Ganje-Fling et McCarthy 1996, 257), mais les abus sexuels sont sans doute parmi les plus nocifs quant au rapport à Dieu. En effet, la plupart des victimes d'abus sexuels souffrent du syndrome de stress posttraumatique (Herman 1992), dont les porteurs démontrent plus de changement dans leurs croyances religieuses que les victimes de traumatisme qui ne souffrent pas dudit syndrome, soit $48 \%$ versus $30 \%$ (Falsetti et al. 2003). C'est que les abus sexuels ont le plus souvent lieu à répétition, durant une période moyenne de cinq ans pour les relations incestueuses (Heary et Solomon 1986, 59), à une époque où se constituent la psyché et la foi de l'enfant. Or, celui-ci se trouve trahi dans son corps et dans son âme, voire dans sa prière, par une personne qu'il aime, qui est censée prendre soin de lui, et à laquelle il identifie (ou a d'abord identifié) la divinité. Si l'éducation religieuse de l'enfant peut l'aider à faire sens de

2. Les données les plus élevées sont celles de Badgley 1984. 
ce qui lui arrive et à le surmonter, elle peut aussi aggraver son sort. L'expérience religieuse des victimes d'abus sexuels illustre les risques et les effets pervers d'un discours religieux qui justifie indirectement la violence et prône la soumission, surtout quand ce discours est adressé aux enfants (Nadeau et Redmond 2002).

Les pages suivantes voudraient rendre compte de l'expérience des victimes d'abus sexuels durant l'enfance face à la Passion de Jésus et présenter de nouvelles voies d'approche qui n'ajoutent pas à leur misère. Nous explorerons donc les liens que font les victimes d'abus sexuels durant l'enfance entre leur expérience et les souffrances du Christ, ainsi que l'impact de cette identification sur leur expérience de foi. Notre réflexion s'organisera donc autour des pôles de l'identification et de l'imitation de la figure de Jésus, qui ont servi tant à soutenir l'espérance des victimes qu'à les écraser davantage, et bien souvent à faire durer les sévices à leur endroit.

La spiritualité et la théologie chrétiennes, particulièrement chez les catholiques, invitent celui ou celle qui souffre à s'identifier au Christ et à offrir sa souffrance pour le salut du monde (Jean-Paul II 1984). Elles invitent aussi le croyant à imiter le Christ, à prendre sa croix et à le suivre (Mc 8,34). Or, si ces thèmes de l'identification et de l'imitation du Crucifié peuvent apporter consolation et espoir, ils peuvent aussi faire problème dans des circonstances où ils contribuent à enfermer ou garder les victimes dans leur état de victimes.

Jossua écrivait: "Celui qui cherche Dieu dans l'histoire se heurte à quelque chose de plus difficile à surmonter que l'invisibilité de Dieu dans les phénomènes: soit la possibilité que Dieu ne soit pas amour. » (1979b, 3) Or, il nous semble que c'est ce qui se passe pour trop de victimes d'abus sexuels durant l'enfance qui font l'expérience de «la possibilité que Dieu ne soit pas amour ", parce qu'il n'intervient pas pour sauver, parce qu'il châtie ou éprouve de façon abusive (Blumenthal 1993), parce qu'il faut se soumettre à sa volonté, endurer et pardonner. C'est ce que les prochaines pages veulent mettre en lumière.

\section{Quand les victimes s'identifient au Crucifié}

L'identification s'inscrit sur deux vecteurs. Sur le premier, la victime s'identifie au Christ souffrant et y trouve force, cette identification donnant sens à la souffrance à travers la participation aux souffrances du Christ ou l'affirmation de la résurrection qui prouve que la souffrance de Jésus n'a pas été vaine, que Dieu n'a pas abandonné Jésus. Sur le second vecteur, 
négatif, la victime s'identifie au Fils abandonné de Dieu ou victime d'un motif divin. L'imitation, quant à elle, s'inscrit sur les vecteurs du devoir: de soumission, de sacrifice et de pardon. Enfin, la victime peut aussi se percevoir responsable, coupable des souffrances de Jésus.

\subsection{S'identifier au Fils abandonné}

La dramatique de l'abandon paraît la plus proche de l'expérience de l'enfant victime d'abus. On sait que l'image de Dieu que porte l'enfant est très marquée par la toute-puissance et la capacité d'intervention divine. Plus l'enfant est jeune lors de l'abus, plus il croit que Dieu devrait le secourir, plus marquées pourront en être son image de Dieu et sa relation à un Dieu qui ne répond pas à ses prières.

I feel like God does not care [...] God has abandoned me and does not answer my prayers. (Fortune 1995, 44)

Each Sunday I'd go to church with my family and hear God loved and was watching over me. Then we'd go home and I'd get abused again and wonder why God didn't protect me from these people who went to church. (Heggen 1993, 43)

One survivor prayed, "God, make Daddy stop doing this to me ». Another asked God not to let her go back into her body after the abuse was over. Since she always did, she felt betrayed by God. (Kennedy 2000, 125)

Many survivors report having felt abandoned by God. "Where was God when I was being abused ?» survivors often ask. Many see God as unfair and unfaithful. They hold God responsible for their abuse. (Heggen 1993, 47)

Bien que dans une perspective différente, le film québécois C.R.A.Z.Y. (2005) de Jean-Marc Vallée illustre bien le désarroi de l'enfant qui prie, sans succès, pour ne pas être homosexuel.

Le théologien mettra en cause l'image de Dieu qui sous-tend la prière de l'enfant et l'éducateur enseignera à l'enfant que le Seigneur exauce autrement ses prières et que ses voies sont insondables. Certains ont trouvé là consolation et espoir. D'autres ont retenu que Dieu protège ceux qu'il aime, qui le prient avec un cœur pur, et qui font sa volonté. Or, cela n'est manifestement pas le cas d'une petite fille ou d'un petit garçon abusés sexuellement... et laissés à eux-mêmes. D'autant que les abus sexuels en milieu familial se poursuivent le plus souvent durant plusieurs années. Quand 
Dieu reste sourd à la prière de l'enfant, quand il n'intervient pas pour elle alors qu'il est si grand, si bon et si puissant, que peut-elle en conclure, elle qui n'a que huit ou dix ans? Que Dieu l'abandonne à son sort? Que Dieu ne l'aime pas, elle? Que Dieu est du côté de celui qui l'agresse jour après jour, semaine après semaine, durant des années? On ne s'étonne pas alors que des victimes et des survivantes rejettent ce Dieu avec l'impression non seulement qu'il les a abandonnées, mais même qu'il a été à la source de leurs souffrances.

As an abused little girl, I prayed and read my Bible both morning and night, and put half of my allowance in the church offering every week, and promised God I'd become a missionary and do anything he asked me to do if he'd just stop my daddy from coming into my room at night. When God didn't answer my prayers, I decided either there wasn't a God or that he was mean and not interested in what happens to little girls. I decided I didn't like God and didn't need a God like him. (Heggen 1993, 44)

Nous y reviendrons dans la section suivante à laquelle nous introduit le troublant Psalm of Anger to a Patriarchal god (Redmond 2000) que nous avons déjà présenté ailleurs (Nadeau 1991; 2005) et dont nous ne citerons ici que quelques versets (Redmond 2000, 33-34):

I trusted you and I believed in you.

When the going got rough, you abandoned me!

I didn't leave you, you left me. [...]

But I will never forget how you abandoned me.

And I will never allow myself to be destroyed by you or your creations

Again!

Where were You when I needed You?

\subsection{S'identifier au Fils, victime de la volonté du Père}

Les enfants sont intelligents - comme les adultes, comme les théologiens - et ils cherchent à comprendre ce qui leur arrive. Leur expérience rejoint celle des croyants dont parle Jon Sobrino lorsqu'il écrit: "The whole question of God finds its ultimate concretion in the problem of suffering. The question rises out of the history of suffering in the world, but it finds its privileged moment on the cross. If the Son is innocent and yet is put to death, 
then who or what exactly is God? " $(1978,224)$ Mais les enfants ne formulent pas ainsi leurs questions, ne peuvent pas prendre ces distances théologiques. Job n'avait pas six ou huit ans, lui. Et combien sont-ils à lui avoir rédigé son texte? De plus, rares sont les enfants élevés chrétiennement qui rejettent Dieu. Qui sont-ils pour rejeter ce super parent Tout-Puissant qui jugera de leur sort éternel ? «... pas un seul cheveu...» (Lc 21,18). L'enfant est sans défense devant un Dieu «qui n’a pas épargné son propre Fils, mais l'a livré pour nous» $(\mathrm{Rm} 8,32)$.

Pour l'âme qui a reçu une éducation chrétienne classique, la souffrance ne saurait être sans raison. Si l'être humain souffre, c'est certes le fruit du péché, mais ce n'est pas tant faute d'intervention que fruit de la volonté de Dieu, soit à titre de punition, d'une épreuve pour la foi ou en vue d'un plus grand bien. La rédemption est au cœur de la foi chrétienne. Or, elle met en scène quelqu'un qui est frappé - et dans plusieurs discours, le Fils par son Père - et qui souffre pour le bien des autres. Pour certaines victimes, le motif de l'abandon se conjugue alors avec celui de la volonté de Dieu. D'autres, habitées de honte et de culpabilité à la suite de leur inceste, ne se croient pas dignes de l'amour divin et en viennent à considérer leur drame comme un châtiment.

I was mad at God-the-Father because he hadn't protected me from Grandpa. He had let me walk right into it. I hadn't deserved that. Something wasn't right. Wasn't He supposed to protect, like a father's supposed to ? I had to be really careful because He saw everything. I didn't understand His almightiness, because he didn't intervene and left me to fend for myself for so long. I had the feeling that I'd fallen for the whole thing. If God could see everything and was so almighty, this would never have happened to me. Or was I a bad child who deserved to be punished? (Imbens et Jonker 1992, 33)

Some survivors really believed God caused the abuse precisely because he seemed to sanction Jesus' death on the cross. If he didn't save Jesus he is not going to save me. (Kennedy 2000, 126)

Others, uncomfortable with such conclusions, decide God isn't the problem - they are. If an all-powerful God allows bad things to happen, it must be punishment they deserve for being bad. (Heggen 1993, 47)

My fear, my childhood training, that tells me that you were on his side? That bad girls go to hell? That your anger is swift and thorough, and poured out on such a one as me? (Foote 1994, 71) 


\section{3. ... et rejeter ce Dieu avec colère}

La tradition propose souvent au croyant de saisir sa souffrance comme une épreuve. Pauvre enfant! Une survivante en colère réagit ainsi:

God is the Father in heaven who does everything for his children because $\mathrm{He}$ loves them. That's what some people say. Well I'm going to tell you exactly what I think of Him. God gave me a mother who didn't want me. She always told me I ruined her life. God gave me a father who raped and abused me for 30 years. God gave me a husband who constantly abused me and my children. God gave my little girl a father who wanted to rape her when she was three and a half. Well thank you very much, God, that you wanted to give me all of this and that you loved me so very much. But God I need nothing more from you, do you hear me? I want absolutely nothing from you just leave me alone, please. (Imbens et Jonker 1992, 209)

Un tel propos marque de part en part le Psalm of Anger to a Patriacal god (Redmond 2000):

You demanded a man kill his own son to prove his faithfulness.

You destroyed a man on a bet and didn't even have the decency to tell him why

You just terrified him into submission.

You even killed your own child!

Don't tell me you couldn't have done things differently.

You seem to delight in putting your children through hell $-[\ldots]$

The best you can do is tell me I need to be forgiven -

\section{FOR WHAT!}

I didn't ask to be born in original sin.

I didn't ask to be raped and beaten and destroyed.

Si c'est par amour et par grâce que le Fils est ressuscité au matin de Pâques, malgré le crime des hommes, peut-on dire que c'est par amour que Dieu éprouva Job ou qu'il permit à Satan de le faire? Le prologue du livre de Job suggère plutôt le contraire. Si Job est éprouvé, ce n'est que dans l'intérêt du Seigneur qui y vérifie sa possibilité d'être aimé (craint) gratuitement. Et tant pis pour Job et les siens! On ne trouve certes pas là le cœur du message du livre de Job auquel le prologue paraît avoir été greffé, mais on comprend le cri que lui adresse, à travers le Psalm of Anger, l'enfant abusée devenue adulte. Au moins est-ce dans l'intérêt des hommes que 
Jésus a été crucifié, ce dont on se serait douté de toute façon, puisque c'est toujours dans l'intérêt des hommes que quelqu'un est sacrifié, torturé et tué. Au moins, Jésus a-t-il librement donné sa vie. Mais n'est-ce pas ce que les sacrifiants affirment de leur victime (Girard 2001; Lempert 2000)?

Plusieurs, mais le plus souvent à l'âge adulte, ressentent donc de la colère contre Dieu. Plus du tiers des victimes interviewées lors d'une étude sur les abus sexuels chez les mormons rapportent avoir vécu une période de colère contre Dieu «because God did not stop their abuse or because God was cast in the image of the "father-perpetrator" " (Gerdes 1996, 54-55). Le plus souvent réprimée chez les enfants, cette colère alors tournée contre soi ne fait qu'accroître leur sentiment de culpabilité. D'autant que, pour l'enfant, Dieu ne saurait être coupable.

\subsection{S'identifier au Christ souffrant}

Heureusement, des survivantes trouvent réconfort dans leur identification au Christ souffrant sur la croix. "The suffering of Jesus on his way to Calvary can be representative of our own struggles. As Jesus experienced, we also know what it means to be unfairly judged, humiliated and even beaten. We can personalize the suffering of Jesus, which can help us to see that we are not alone in our pain.» (Flaherty 1992, 74) La prière d'une victime sensible à une figure de Christa affirme l'effet positif d'une telle identification à l'âge adulte. Nous en reproduisons le début et la fin:

\section{O God}

Through the image of a woman

Crucified on the cross

I understand at last. [...]

You were not ashamed of your wounds.

You showed them to Thomas

As marks of your ordeal and death.

I will no longer hide these wounds of mine.

I will bear them gracefully.

They tell a resurrection story. (Fortune 1987, 68-69) 
Une autre survivante en témoigne aussi lorsqu'elle propose un chemin de croix à l'intérieur d'une démarche de résolution de l'abus. À titre indicatif, nous en retenons les thèmes suivants (Flaherty 1992, 74-76):

$-2^{\mathrm{e}}$ station: Jésus prend sa croix

Quel fardeau a été ma croix?

$-5^{\mathrm{e}}$ station: Simon aide Jésus

À l'enfance, qui m'a apporté de l'amour, de la tendresse?

$-6^{\mathrm{e}}$ station: Véronique aide Jésus

Qui m'a aidée pendant ma démarche de résolution de l'abus?

$-10^{\mathrm{e}}$ station: Jésus est déshabillé

À quel moment de l'abus ai-je ressenti de l'humiliation?

$-11^{\mathrm{e}}$ station: Jésus est crucifié

À quel moment de l'abus ai-je ressenti de la douleur physique?

- 14 e station: Jésus est enterré

Quelles parties de moi restent enterrées ou cachées à cause de l'abus?

Ici encore, nous sommes devant l'expression d'une adulte qui a longuement cheminé dans la résolution spirituelle de son expérience d'abus. La situation est bien différente pour les enfants et nous avons trouvé très peu de citations montrant qu'ils aient trouvé réconfort dans leur identification au Crucifié. Peut-être s'agit-il davantage d'une démarche d'adulte que d'enfant encore soumis aux abus, plus impressionné par sa condition et moins bien nanti de ressources psychoaffectives et théologiques que l'adulte qui a survécu aux abus et est en voie de se reconstruire.

Quelques adultes rappellent néanmoins l'expérience religieuse de leur enfance. Ruth, par exemple, se souvient de sa complicité avec l'enfant Jésus qu'elle priait en se disant que "he was a child too and that maybe a child would see another child's pain more easily» (Jamieson 1995, 60). Une autre survivante se rappelle du soutien du Christ: "The sexual abuse in my childhood was severe, and often my world shifted into cycles of unreality. Strangely enough, the one reality I can remember that always held (even when I was a little child) was Christ. I don't know how this happened or why especially to me - all I know is that when things were most confusing, he was there.» (Hancock et Burton 1987, 84)

Pour d'autres enfin, ces souvenirs restent douloureux: "When I was being abused I saw a crucifix on the bedroom wall — if I go into a Church and see a crucifix I'm right back in my bedroom. » (Kennedy 2000, 161) 


\section{Les injonctions de l'imitation}

Imposée, l'identification se confond avec l'injonction d'imitation et devient une arme entre les mains des bourreaux. Poling en témoigne lorsqu'il rapporte, à partir de sa pratique de relation d'aide auprès de victimes et d'agresseurs: «Perpetrators have often told their victims that they should not complain of their abuse because their suffering is like that of Jesus and they will be rewarded in the future. » $(1996,146)$ Malheureusement, il n'y pas que les abuseurs qui tiennent ce discours, qui marque au contraire toute la tradition.

\subsection{Obéir et porter sa croix}

I kept praying the Lord will give me His ability to withstand the pain [...]. After all, Jesus requires those who follow him to deny themselves and pick up their cross. I guess this [the sexual abuse] is my cross to bear.

(Manlowe 1995, 63)

L'imitation du Christ et les injonctions à la patience et à la soumission qui la déterminent largement constituent un motif qui peut devenir particulièrement lourd pour les victimes d'abus sexuels. Si l'identification avec le Christ de la Passion peut s'avérer spontanée, l'imitation relève largement du devoir, devoir de porter sa croix et de suivre le Christ dans la souffrance. Or, la figure de Jésus majoritairement promue est celle du Fils qui se soumet à la volonté de son Père, obéissant jusqu'à la mort, et la mort de la croix. La soumission de Jésus apparaît alors comme un cauchemar, un concept terrifiant pour plusieurs qui en viennent à craindre la volonté divine (Imbens et Jonker 1992, 213).

Le message salutaire de la croix se trouve donc compliqué par le fait que le Christ y a souffert dans une obéissance totale à son Père comme en témoigne le récit de Gethsémani. L'enfant qui reçoit une éducation chrétienne apprend que le sacrifice et l'obéissance, voire la souffrance sont des vertus indispensables à tout bon chrétien, voire des vertus identitaires du chrétien (Redmond 1989). Poursuivi par ses ennemis, rongé par l'angoisse, arrêté et crucifié, Jésus se soumet à la Volonté de Dieu, son Père. Il accepte la souffrance, reste le plus souvent silencieux, ne se rebelle pas, ne demande 
pas d'aide... et pardonne. Or, il est LE modèle, aussi bien pour les faibles que pour les forts, et d'abord pour les enfants, sans tenir compte de leur contexte. Dans ce modèle, la souffrance est acceptable, voire désirable. "The priest said I should rejoice in my sufferings because they bring me closer to Jesus. He said, "Jesus suffered because he loved us." He said, "If you love Jesus, accept the beatings and bear them gladly, as Jesus bore the cross [...]." I recognized that Christianity had taught me that sacrifice is the way of life." (Brock et Parker 2001, 6) "My mother always said to me that if I made my bed hard, I would have to lie in it. This one is pretty hard, all right. Is this just my cross to bear?» (Fortune 1995, 18)

Si de tels discours peuvent faciliter la victimisation des enfants, comme des adultes, le théologien et plusieurs croyants savent que l'imitation du Christ peut mener à se tenir debout devant l'adversaire, à tenir bon pour sa foi, etc. L'obéissance de Jésus constitue un riche motif théologique dont Richard Bergeron (1976), par exemple, a bien fait ressortir la complexité. Ainsi, l'obéissance à Dieu est souvent tout le contraire du silence et de la soumission aux hommes. Mais ce n'est pas ce qu'on apprend habituellement aux enfants. Dans une culture sociale et religieuse qui affirme l'autorité des parents sur les enfants - à laquelle correspond celle des clercs sur les laïcs - , les thèmes de l'imitation du Christ et de sa soumission à la volonté de son Père sont le plus souvent au service de l'obéissance familiale, religieuse et politique, voire de la domination qu'ils fondent souvent sur le plan religieux. Ce Père auquel Jésus obéit jusqu'à la mort de la croix, et que préfigurait Abraham partant sacrifier son fils à la volonté d'un songe, c'est aussi celui de l'enfant... dont les parents sont «les représentants sur la terre». Mais qui apprend à ses enfants à résister dans le cadre familial? Qui, sauf exceptions récentes, apprend aux petites filles à résister aux hommes de la famille? Qui apprend aux enfants à résister à l'autorité injuste?

Dans quelque culture que ce soit, l'obéissance et la soumission apparaissent comme des vertus religieuses fondamentales. En témoigne le Petit catéchisme de Québec dont l'obéissance était le pivot (Dussault 1972; Raymond 1983). L'enfant doit obéir. Il assume ou doit assumer que ce que l'adulte lui demande est juste et que le refus de s'y soumettre implique la perte de l'amour de l'adulte, voire de l'amour de Dieu. Combien d'entre nous se sont confessés d'avoir désobéi à leurs parents? 
2.2 «Père, pardonnez-leur...»

The Bible says that I must forgive him. But I don't feel very forgiving. How can I forgive all that he did? (Fortune 1995, 52)

Passion, sacrifice et pardon sont intimement liés dans l'esprit des victimes comme ils le sont dans la théologie classique. C'est bien pour le pardon de nos fautes que Jésus a souffert. Et quelle valeur chrétienne aurait le sacrifice des victimes si à l'instar de Jésus elles ne pardonnaient pas à leurs bourreaux $^{3}$ ? La question se pose d'autant plus pour la victime qu'on lui a dit qu'elle est - et qu'elle se sent - en grand besoin de rédemption de ses fautes sexuelles! «Pardonnez et l'on vous pardonnera » affirme l'Évangile (voir Mt 6,14-15; Mc 11,25-26). Refuser de pardonner apparaît comme une faute, une faute de plus (Nadeau 2003)! Une faute que Dieu ne pardonnerait pas puisque «Si vous pardonnez aux hommes leurs offenses, votre Père céleste vous pardonnera aussi; mais si vous ne pardonnez pas aux hommes, votre Père ne vous pardonnera pas non plus vos offenses " (Mt 6,14-15). Et puis - et cela fait partie du contexte de lecture de la Passion - nous prions chaque jour notre Père de nous pardonner «comme nous pardonnons»!

L'épisode suivant est éloquent même s'il n'a pas de rapport explicite avec la Passion. "One incest survivor was told by her mother as her father lay dying: "If you don't forgive your father, he'll burn in Hell forever!" ” Et voilà, perversement, que la victime devient responsable du salut éternel de son agresseur. Comme le signale Keene, qui rapporte l'anecdote: "Typically the forgiveness dictum gets turned against the victim: a woman's father may have raped her and she may have spent her life recovering from his violation, but if she's not ready to forgive, then her hardheartedness is her fault—and maybe God won't forgive her. » (1995, 32-35)

Autre injonction pernicieuse: celle de ce père qui, après avoir abusé de son fils, "would get down on his knees and also tell his son to pray with him to ask forgiveness for what they had both done» (Kennedy 2000, 124131 , nos italiques). Et voilà que la prière se transforme en outil de culpabilisation et de manipulation, d'autant plus vive que la demande de pardon

3. Si Jésus n'a pas pardonné lui-même à ses bourreaux, il a néanmoins prié son Père de le faire. 
de l'agresseur s'articule souvent autour de menaces et de l'obligation du silence de la victime, qui constitue un élément majeur de la dramatique de l'inceste.

\section{3 «Livré pour nos péchés»}

He started abusing me when I was five years old. This was when I was beginning my religious training which taught me that women were vessels of sin. It was my sin of incest that made him [Jesus] hang on the cross.

(Redmond 1989, 77)

Nous passerons plus rapidement sur ce dernier thème, moins présent dans la littérature. On sait que la théologie classique résume la Passion en affirmant que, selon la volonté de Dieu, Jésus a souffert et est mort pour notre salut, en rémission des péchés. "He was wounded for our transgressions, crushed for our iniquities; by His wounds, we are healed » rappelle justement l'exergue du film de Gibson citant Is $53^{4}$. Or, l'éducation chrétienne fait en sorte que les victimes d'inceste se sentent particulièrement coupables aux yeux de Dieu étant donné la nature de «leur» faute. La victime d'abus sexuels se sent salie, irrémédiablement souillée. La morale sexuelle impitoyable de son éducation religieuse la comble de honte et de culpabilité, particulièrement en cas d'inceste. Quand l'enfant apprend aussi que ses fautes sont la cause des souffrances du Christ, la croix, qui est pour nous symbole de rédemption et de salut, peut alors devenir le signe de sa culpabilité et de sa condamnation.

\section{Reconstruire le discours de la Passion}

Heureusement, même s'ils ne se rendent pas toujours jusqu'aux enfants, d'autres discours théologiques critiquent ces images de Dieu et permettent

4. On trouve des échos de ce texte dans le Nouveau Testament ( $R m$ 4,25; Ep 5,2; $\mathrm{Ga} 2,20 ; 1 \mathrm{Co} 15,3 ; 1 \mathrm{P} 2,24)$. Notons au passage le flou qui entoure les références à cet exergue. Alors que les articles anglophones renvoient le plus souvent et avec justesse à Is 53,5, les articles francophones (par exemple Gignac 2004) renvoient plutôt à Is 53,4: "Ce sont nos souffrances qu'il portait et nos douleurs dont il était chargé » - ce qui place le film et la Passion sous une toute autre interprétation. 
de saisir autrement Dieu et la Passion. Nous en exposerons rapidement quelques-uns.

\subsection{Une critique nécessaire}

Nous avons constaté à quel point le discours traditionnel chrétien peut être dur pour les victimes d'abus sexuels et particulièrement d'inceste. Loin de contribuer au salut des victimes ou de leur apporter réconfort, plusieurs éléments du discours traditionnel sur la Passion leur imposent un fardeau additionnel: valeur infinie de l'obéissance et de la souffrance jusqu'à la mort, image du père divin qui abandonne (ou même châtie) son fils, obligation de pardonner, péché et culpabilité (particulièrement sur le plan sexuel, on le sait par ailleurs), etc.

Ce qui est en jeu ici, c'est la capacité de l'enfant abusée sexuellement et donc profondément marquée de trouver la force vivifiante de la Passionmort-et-résurrection de Jésus Christ. Comment une petite fille abusée sexuellement par son père ou une autre figure importante dans sa vie peutelle avoir péché? Comment peut-elle être responsable des horreurs qu'on lui impose ? Comment est-elle tenue à l'impératif de pardonner ? Pourra-t-elle encore prier Dieu, son Père au ciel qui l'abandonne et qu'elle identifie souvent avec son père sur la terre? Et comment peut-elle réagir devant un discours qui propose la soumission de Jésus en modèle à l'enfant? Certes, la théologie a bien des réponses à ces questions, mais elles restent le plus souvent insatisfaisantes et, rappelons-le, car c'est majeur, l'enfant n'a pas les ressources de l'adulte ni celles de la théologie.

Le théologien et la théologienne sont plus au fait des difficultés et des apories du langage de la croix et souvent plus habiles à en prendre distance. Certains, tel Guardini s'acheminant vers la mort (Rahner 1983, 208), avouent néanmoins leur incompréhension profonde devant la croix. D'autres, au contraire, professent y voir clair, tel Mouw qui rappelle que l'œuvre de la croix relève de la Trinité et qu'on ne saurait y opposer le Père et le Fils: "The very same God who pours out the divine wrath is the one who experiences the wrathful forsakenness of divine abandonment. God, in the unity of the divine being, is both the violated One and the One who counts that violatedness as satisfying the demands of eternal justice." $(2003,171)$

L'affirmation, comme sa rhétorique, est traditionnelle. Elle met en valeur qu'il s'agit là d'une dynamique interne à la Trinité, comme en parlait déjà bien mieux Le Dieu crucifié de Moltmann (1978). Mais Mouw va 
beaucoup plus loin et avec nettement moins de sensibilité que Moltmann qui évite le discours de la colère divine: "God, écrit Mouw, is both the violated One and the One who counts that violatedness as satisfying the demands of eternal justice." Le plus troublant, c'est qu'il prétend ainsi répondre aux objections de Joanna Carlson Brown citées au début de notre texte, objections qu'il a bien rapportées, mais auxquelles il n'a manifestement rien compris. Ne voit-il pas que ce que dénonce Brown, c'est justement ce discours d'acceptation dans l'amour, ce discours qui justifie la souffrance et la profanation (violatedness — si proche du français «viol»)! Et comment dire aux victimes que «la profanation du Fils innocent satisfait aux exigences de la justice éternelle» toujours liée à la divine wrath? Le cœur de la foi chrétienne serait donc la relation entre un Fils victime de la nécessité de logiques pénale et courtisane, et un Père qui en compte les mérites, fût-il le même Dieu qui en souffre aussi! Le pire, c'est que Mouw n'a même pas l'excuse d'ignorer le problème auquel il prétend justement répondre! Pire encore, car cela montre l'étendue des dégâts, il représente bien la tradition classique encore la plus répandue! Certes, sur la croix, Dieu, le Fils, ne répond pas à la violence par la violence. Mais il y apparaît néanmoins soumis aux lois de celle-ci, même si son attitude, en deçà du discours reçu, peut s'avérer subversive. Trop souvent, l'abandon du Fils considéré comme non-violence - apparaît relever des impératifs supérieurs d'une rétribution accomplie dans la violence. Et y a-t-il plus violent que le «wrath of God» illustrée aussi bien par l'enfer des Rédemptoristes que par celui de Jonathan Edwards? Certes, ce discours n'est pas d'abord destiné aux enfants! Malheureusement, c'est bien celui qu'ils apprennent avec la Passion. De toute évidence, Mouw confirme les pires expériences des victimes.

S'il faut saluer l'effort de ces théologies pour rendre compte du salut, on ne peut que souhaiter que leur mécanique relève d'une époque théologique révolue. Ces constructions qui pouvaient être signifiantes à certaines époques font aujourd'hui problème et peuvent même s'avérer pires pour les victimes que les questions auxquelles elles veulent répondre. Pires «parce que faibles. Ou parce que reposant sur des fondements insuffisamment critiqués. Ou encore parce qu'apparemment insensibles à l'expérience en cause [...]. Ou encore parce que plus scandaleuses dans leur principe que la difficulté qu'elles prétendent surmonter. »(Jossua 1979a, 1) Parmi ces principes, celui selon lequel «la fin ne justifie pas les moyens». Mauvaises enfin, répétons-le, à cause de leur usage mortifère pour prôner la résignation et tuer la révolte contre le mal (Tilley 1991). C'est ainsi que Brock 
(1989) et bien d'autres depuis Mary Daly considèrent que la glorification de la soumission et de l'obéissance constitue un abus de la christologie au profit de ceux qui détiennent le pouvoir.

\subsection{D'autres voies}

Heureusement, le vingtième siècle a vu émerger des critiques de ces théologies traditionnelles et proposer d'autres approches. Elles sont nombreuses et nous ne pouvons pas les signaler toutes, fût-ce aussi brièvement.

- La position qui est sans doute la plus populaire actuellement et dont Moltmann apparaît comme le chef de file critique l'image d'un Dieu apathétique et affirme que Dieu souffre avec nous.

- Une autre, qui relève surtout de Metz et de la théologie de la libération latino-américaine, insiste sur la résurrection et sur son potentiel d'espérance pour les crucifiés de ce monde (Sobrino).

- D'autres approches situent la souffrance dans le plan de Dieu comme une dimension du combat contre le mal et l'injustice ou, avec la théologie du procès (process theology), une dimension du développement (procès) de Dieu et de la création.

- Des approches féministes, qui ont largement marqué notre réflexion, proposent une figure féminine de la divinité, souvent holistique, une figure qui a souvent aidé les survivantes adultes, mais qui paraît plus éloignée des enfants victimes pour lesquels la relation à la mère est souvent aussi trouble que celle avec le père.

- Une autre approche saisit la Passion, la mort et la résurrection de Jésus comme témoignant de l'incompréhensibilité de Dieu (Rahner).

- Une approche plus radicale, à laquelle nous avons déjà fait écho avec Brock, rejette la notion dogmatique de souffrance rédemptrice et présente la croix du Christ comme le symbole par excellence de la vulnérabilité et de la résistance de l'humain et de Dieu.

- Une approche éthique soutient que Dieu n'a pas d'autres mains, d'autres yeux, d'autres pieds que les nôtres, une position particulièrement exigeante qui ne soutiendra les victimes que si les croyants la prennent vraiment au sérieux.

Il est impératif de développer une «christologie» qui refuse le statut de volonté divine à la souffrance et, en conséquence, à la violence dont elle est issue. Poling considère la reconstruction du symbole christologique par les 
personnes opprimées comme une tâche herméneutique - nous dirons " théologique» - cruciale (1996, 149). Or, cette reconstruction constitue un processus délicat étant donné la diversité des contextes d'expérience et de réflexion, de même que la difficulté à dire le sens du salut à notre époque. Peut-être est-ce aux victimes qu'il faudrait le demander. Mais même alors il varie - mais faut-il qu'il soit univoque? Les unes y voient l'arrêt des actes perpétrés contre elles; d'autres y voient la possibilité de "passer à travers » et d'y survivre; d'autres, l'espérance d'un monde meilleur. Si nous ne pouvons entreprendre ici ce travail de reconstruction herméneutique, quelques remarques s'imposent néanmoins:

- Un Dieu patriarcal et seigneurial (féodal) est aujourd'hui inacceptable (McFague 1987).

- De la pratique de Jésus, il faut souligner la dynamique de résistance bien davantage que celle de soumission.

- Il faut être prudent quant aux affirmations sur la valeur de la souffrance et peut-être distinguer, comme Fortune (1983), entre la souffrance qui résulte d'un projet et d'un risque assumé, et celle qui résulte de l'arbitraire et de l'abus du pouvoir (Blumenthal 1993). De plus, il faut aussi distinguer la souffrance de l'enfant et celle de l'adulte.

- De même, il importe de distinguer l'éducation religieuse de l'enfant de celle de l'adulte.

- Quant à la faute, l'enfant doit savoir qu'elle appartient ici à l'adulte et qu'elle ne saurait le priver de la présence et de l'amour divins, ce qui est certes difficile étant donné la dynamique psychique de l'abus sexuel.

Dieu est mystère. Et son agir aussi. Mais si Dieu agit, c'est du côté de la grâce, du salut, de la résurrection et de la vie. Pas du côté de l'injustice et de la torture. Si l'on peut reconnaître la présence de Dieu à la croix et éventuellement y trouver réconfort, c'est à la Résurrection que se manifestent sa signature, sa volonté et sa puissance de vie. C'est l'espoir de cette résurrection, l'espoir d'une vie différente, d'une vie libérée de l'oppression et sans violation qui a gardé vivantes bien des victimes. Et c'est là qu'il faut chercher. Ce qui sauve, c'est la résurrection, la grâce de la résurrection. La Passion s'inscrit dans la logique d'un projet risqué et assumé, Jésus s’y avérant victime de la violence religieuse et politique. La résurrection n'est pas une justification rétrospective de la violence des hommes et de la souf- 
france qui en est issue, mais l'affirmation qu'une vie nouvelle reste possible pour les victimes.

Contrant l'utilisation de la Passion et de la croix pour justifier ou encourager une résignation passive face à la violence et à l'abus, on y voit aujourd'hui le symbole de la résistance. Dans une réflexion sur la morale chrétienne, $\mathrm{LeDu}$ (1976) signalait déjà comment le Christ avait subverti sa Passion, s'y tenant bien autrement que comme victime passive et résignée. Plus récemment, Brock (1993) rappelait que la détermination et la résistance sont aussi caractéristiques de la pratique de Jésus et proposait d'imiter ce Jésus prophétique et rebelle plutôt que le Jésus obéissant et docile. Globalisant le propos, Poling considère que

Jesus' resistance to evil is a manifestation that assures us that human resistance to evil can be trusted, because it is the image of God in relation between the divine and the human (and so the true nature of both divine and human). Jesus resisted the evil of his day, even to death on a cross, and thus raises to ontological status human resistance to evil. (Poling 1996, 157)

Pour lui, et notre expérience le corrobore largement, les victimes et survivantes d'abus sexuels font partie des personnes et communautés qui incarnent aujourd'hui cette résistance.

Comme plusieurs chrétiens, nous avons été tentés de regretter que les notions d'espérance, de joie, de résurrection soient trop souvent absentes du discours sur la Passion du Christ, un discours qui s'arrête souvent au Vendredi saint et oublie le mystère de la résurrection. Mais il faut aller audelà de ce regret et porter attention au type d'articulation que le discours sur la Résurrection opère avec la Passion et la souffrance de Jésus. Si la Résurrection est la sanction divine pour l'obéissance et les souffrances du Fils, on ne règle rien à la valoriser davantage. Si la Résurrection est un acte gratuit de Dieu qui redonne toujours vie, si elle est l'acte d'un Dieu qui, loin de sanctionner positivement la violence des hommes, s'inscrit en faux contre elle, c'est alors d'autre chose qu'il s'agit. Non pas d'un lien de cause à effet avec la soumission du Fils à la violence des hommes, mais un lien de retournement, de re-surrection. Émerge alors une figure de la divinité différente de celle qui dominait la première partie de notre réflexion. Une figure proprement pascale, dont nos lectures et les entrevues que nous menons auprès de survivantes indiquent que certaines y trouvent effectivement une dynamique d'espérance et de résistance. C'est ainsi que quelques survivantes relient leur propre expérience au mystère pascal, témoignant de ses possibilités pratiques pour elles. "Good Friday is essential but it isn't an end in 
itself. The point is to move into the Easter Sunday experience and to live from that. It was through both Good Friday and Easter that I learned who God is - and who I am.» (Jamieson 1995, 92) Ce passage du Vendredi saint à Pâques est difficile, on le sait. Et elles le savent bien davantage encore. D'autant que le Vendredi saint, c'est souvent la souffrance de se sentir abandonnées par Dieu, de devoir faire le deuil du Dieu qui n'a pas sauvé, le deuil d'une certitude ébranlée par l'expérience. Or, des victimes, des survivantes ont finalement découvert, avec joie et surprise, non seulement qu'il était possible et correct de douter dans de telles circonstances, mais que Dieu leur était présent même dans ces temps de doute et d'errance spirituelle. Comme l'écrit l'une d'elles: "What a delight to discover that God does not demand unquestioning belief, but rather stands with us in the times of doubting too.» (Foote 1994, 10) Une découverte libératrice pour plusieurs et qui constitue un pas majeur dans la réhabilitation (spirituelle) des victimes.

\section{Références}

BADGLey, R.F. (1984), Infractions sexuelles à l'égard des enfants / rapport du Comité sur les infractions sexuelles à l'égard des enfants et des jeunes, Ottawa, Approvisionnements et Services Canada.

Bergeron, R. (1976), Obéissance de Jésus et vérité de l'homme: une interpellation, Montréal, Fides.

Blumenthal, D.R. (1993), Facing the Abusing God: A Theology of Protest, Louiseville, Westminster / John Knox Press.

Brock, R.N. (1989), "And A Little Child Will Lead Us: Christology and Child Abuse ", dans J.C. Brown et C.R. Bohn, dir., Christianity, Patriarchy and Abuse. A Feminist Critique, New York, Pilgrim Press, p. 42-61.

(1993), "Losing your Innocence but Not Your Hope », dans M. Stevens, dir. Reconstructing the Christ Symbol. Essays in Feminist Christology, New York, Paulist Press, p. 30-53.

Brock, R.N. et R.A. Parker (2001), Proverbs of Ashes: Violence, Redemptive Suffering, and the Search for What Saves Us, Boston, Beacon Press.

Brown. J.C. et R.A. Parker (1989), «For God so Loved the World? », dans J.C. Brown et C.R. Bohn, dir., Christianity, Patriarchy and Abuse. A Feminist Critique, New York, Pilgrim Press, p. 1-30. 
Dussault, G., s.j. (1972), «La religion de l'ordre... et après ? ", Relations, 377, p. 330-334.

Falsetti, S.A., P.A. Resick et J.L. Davis (2003), "Changes in Religious Beliefs Following Trauma ", Journal of Traumatic Stress, 16/4, p. 391398.

Flaherty, S.M. (1992), Woman, Why Do You Weep? Spirituality for Survivors of Childhood Sexual Abuse, New York / Mahwah, Paulist Press.

Foote, C.J. (1994), Survivor Prayers. Talking with God about Childhood Sexual Abuse, Westminster, John Knox Press.

Fortune, M.M. (1983), Forgiveness: The Last Step, Lexington / Toronto, Lexington Books.

(1987), Keeping the Faith: Guidance for Christian Women Facing Abuse, San Francisco, Harper.

(1991), Violence in the Family: A Workshop Curriculum for Clergy and Other Helpers, Cleveland, Pilgrim Press.

(1995), Keeping the Faith: Guidance for Christian Women Facing Abuse, San Francisco, Harper.

Ganje-Fling, M.A. et P. McCarthy (1996), "Impact of Sexual Abuse on Client Spiritual Development: Counseling Implications ", Journal of Counseling and Development, 74/3, p. 253-258.

Ganzevoort, R.R. (1994), "Crisis Experiences and the Development of Belief and Unbelief », dans J. Corveleyn et D. Hutsebaut, dir., Belief and Unbelief: Psychological Perspectives, Amsterdam / Atlanta, Rodopi (International Series in the Psychology of Religion 3), p. 21-36.

Gebara, Y. (1999), Le mal au féminin. Réflexions théologiques à partir du féminisme, Paris, L'Harmattan (Religion et sciences humaines).

Gerdes, K.E. (1996), "Adult Survivors of Childhood Sexual Abuse: The Case of Mormon Women ", Affilia: Journal of Women and Social Work, 11/1, p. 39-60.

Gignac, A. (2004), «La Passion selon Gibson. Une œuvre réductrice et névrosée », Le Devoir (28 janvier), p. E1.

Ginter, J. (2004), Resiliency among Sexual Abuse Survivors within the Christian Church, communication au congrès annuel du Council of Societies for the Study of Religion, non publiée. 
Girard, R. (2001), Celui par qui le scandale arrive, Paris, Desclée de Brouwer.

Hancock, M. et K. Burton Mains (1987), Child Sexual Abuse. Hope for Healing, Wheaton, Harold Shaw.

Heary, B.B. et R.S. Solomon (I986), Child Maltreatment and Paternal Deprivation, Levington, Lexington Books.

Heggen, C.H. (1993), Sexual Abuse in Christian Homes and Churches, Scottdale / Waterloo, Herald Press.

Herman, J.L. (1992), Trauma and Recovery. The Aftermath of Violence from Domestic Abuse to Political Terror, New York, Basic Books.

Imbens, A. et I. Jonker (1992), Christianity and Incest, Minneapolis, Fortress Press.

Jamieson, H. (1995), Childhood Sexual Abuse and the Development of Women's Spirituality. Educational Psychology, Edmonton, University of Alberta.

Janoff-Bulman, R et C. Frants (1997), "The Impact of Trauma on Meaning: From a Meaningless World to a Meaningful Life», dans M. Power et C. BREwin, dir., The Transformation of Meaning in Psychological Therapies, Londres, Wiley.

JeAn-Paul II (1984), Le sens chrétien de la souffrance humaine / lettre apostolique Salvifici Doloris, Montréal / Paris, Fides / Cerf.

Jordan, M.R. (1995), "A Spiritual Perspective on Trauma and Treatment", National Center for Post-Traumatic Stress Disorder Clinical Quarterly, 5/1, p. 9-10.

Jossua, J.-P. (1979a), Discours chrétiens et scandale du mal, Paris, Chalet. (1979b), Un homme cherche Dieu, Paris, Cerf.

Keene, F. (1995) «The Politics of Forgiveness: How the Christian Church Guilt-Trips Survivors ", On the Issues: The Progressive Women's Quarterly, 4/4, p. 32-35.

Kennedy, M. (2000), "Christianity and Child Sexual Abuse. The Survivor's Voice Leading to Change », Child Abuse Review, 9, p. 124-131.

LeDu, J. (1976), La tentation de Jésus ou L'économie des désirs, Saint-Brieuc, SOFEC.

Lempert, B. (2000), Critique de la pensée sacrificielle, Paris, Seuil. 
Lifton, R.J. (1988), «Understanding the Traumatized Self: Imagery, Symbolization, and Transformation ", dans J.P. Wilson et al., Human Adaptation to Extreme Stress: From the Holocaust to Vietnam, New York, Plenum, p. 7-31.

Manlowe, J.L. (1995), Faith Born of Seduction: Sexual Trauma, Body Image, and Religion, New York / Londres, New York University Press.

McFague, S. (1987), Models of God. Theology for an Ecological, Nuclear Age, Philadelphie, Fortress Press.

Moltmann, J. (1978²) [1974, allemand 1972], Le Dieu crucifié. La croix du Christ, fondement et critique de la théologie chrétienne / trad. par B. Fraigneau-Julien, Paris, Cerf / Mame (Cogitatio fidei 80).

Mouw, R.J. (2003), "Violence and the Atonement», dans K.R. Chase et A. Jaсовs, Must Christianity Be Violent?, Grand Rapids, Brazos Press.

Nadeau, J.-G. (1991), "Éducation chrétienne et réaction à l'inceste », Prêtre et Pasteur, 94/5, p. 276-286.

- (2003), «Le pardon, une affaire de justice et de pouvoir », Scriptura: Nouvelle série, 5/2, p. 47-60.

(2005) «Bricoler pour survivre. La mémoire et l'identité religieuse des victimes d'abus sexuels ", dans R. VAN DEN BRANDT et M. KOOPMANThurlings, dir., Bricoler la mémoire. La théologie et les arts face au déclin de la tradition, Paris, Cerf (en voie de parution).

Nadeau, J.-G. et S.A. Redmond (2002), "Le "Dieu des victimes" reconsidéré à partir de la dynamique des abus sexuels en milieu chrétien ", dans P. NoËL, dir., Violence, mondialisation et religion / actes du $39^{\mathrm{e}}$ congrès de la Société canadienne de théologie (octobre 2002), Montréal, Fides (en voie de parution).

Overcash, W.S., L.G. Calhoun, A. Cann et R.G. Tedeschi (1996), "Coping with Crises: An Examination of the Impact of Traumatic Events on Religious Beliefs ", The Journal of Genetic Psychology, 157/4, p. 455-464.

Pargament, K.I., H. Olsen, B. Reilly, K. Falgout, D.S. Ensing et K. Van Haitsma (1992), "God Help Me (II): The Relationship of Religious Orientations to Religious Coping with Negative Life Events ", Journal for the Scientific Study of Religion, 31/4, p. 504-513.

Poling, J.N. (1996), Deliver Us From Evil. Resisting Racial and Gender Oppression, Minneapolis, Fortress Press. 
Rahner, K. (1983), "Why Does God Allow Us to Suffer? ", Theological Investigations, 19, p. 194-208.

Raymond, G. (1983), "Quelles catéchèses ? Quelles éthiques ?", Communauté chrétienne, 132, p. 580-595.

Redmond, S.A. (1989), "Christian "Virtues" and Recovery from Child Sexual Abuse", dans J.C. Brown et C.R. Bohn, Christianity, Patriarchy and Abuse: A Feminist Critique, New York, Pilgrim Press, p. 70-88.

(2000), «Psalm of Anger to a Patriarchal god », Théologiques, 8/2, p. 33-34. D'abord publié sous le titre "Confrontation Between the Christian God and an Abused Child: Twenty-Five Years Later ", dans Church Council on Justice and Corrections, dir., Family Violence in a Patriarchal Culture: A Challenge to Our Way of Living, Ottawa, Canadian Council on Social Development, 1989.

Sobrino, J. (1978), Christology at the Crossroads: A Latin American Approach, Maryknoll, Orbis Books.

(1986), Jésus en Amérique latine. Sa signification pour la foi et la christologie, Paris, Cerf (Cogitatio Fidei 140), p. 245-261.

Tilley, T.W. (1991), The Evils of Theodicy, Washington, DC, Georgetown University Press.

VAn Der Kolk, B.A. (1987), Psychological Trauma, Washington, DC, American Psychiatric Press.

Weaver, A.J., H.G. Koenig et F.M. Ochberg (1996), «Posttraumatic Stress, Mental Health Professionals and Clergy: A Need for Collaboration, Training and Research », Journal of Traumatic Stress, 9/4, p. 847-856.

\section{Résumé}

Les croyances religieuses offrent en principe des ressources pour faire face aux événements traumatisants, mais il y a aussi des cas où elle en accroît le traumatisme ou en retarde la résolution. L'article rend compte de l'expérience de victimes d'abus sexuels durant l'enfance face à la Passion de Jésus. La réflexion s'organise autour des pôles de l'identification et de l'imitation de la figure de Jésus. Non seulement le discours traditionnel chrétien peut-il s'avérer délétère pour les victimes, mais les nombreuses approches critiques développées au vingtième siècle s'avèrent fréquemment insuffisantes pour les victimes. Il demeure donc impératif de développer une christologie qui refuse le statut de volonté divine à la souffrance et à la violence dont elle est souvent issue. 


\begin{abstract}
Religious beliefs theoretically offer resources to help face traumatizing events, but they can also increase the trauma or delay its resolution. This article accounts for the experience of victims of childhood sexual abuse with respect to the Passion of Jesus. It is structured around the poles of identification with and imitation of the figure of Jesus. Not only can traditional Christian discourse prove to be harmful for the victims, but the many critical approaches developed during the twentieth century also frequently prove unsatisfactory for the victims. Thus, it remains crucial to develop a Christology that refuses the statute of divine will to suffering and to the violence from which it often results.
\end{abstract}

